\title{
Editorial
}

\section{Statistical Estimations in Complex Problems}

The current issue is devoted to statistical estimations in complex problems. Several novel methods of solving different applied problems are discussed, and some unique approaches of statistical analysis are introduced. Eight theoretical and applied papers devoted both to modern statistical estimations in multi-person multi-criteria decisions and other complex problems, statistical methods and their application to different decision making, optimization and operations research problems, particularly, for continuous and discrete systems with single and multiple criteria are presented.

The issue starts with a research work "A study on estimation of reliability of a coherent system under field condition" by Prof. Debasis Bhattacharya from Santiniketan and Prof. Soma Roychowdhury from Calcutta, India. Reliabilities of the components of an engineered system are estimated usually under a laboratory set up while the system may be installed to work under a different, the so called "field" condition. The cause of the difference may be attributed to a random variable, called an "environmental" variable, the presence of which makes the component failure-times dependent. If the system reliability does not account for such conditions, there would be an upward or downward bias in estimation. The authors study the effect of an environmental variable on the reliability estimation of a complex coherent system, and find out the conditions under which the system performance is optimized. A simulation study has been done to see the crossing behavior of the reliability functions under laboratory and field conditions.

A team of authors leaded by Prof. Hui Gong from Valparaiso, USA, and accompanied by Profs. Aerambamoorthy Thavaneswaran and Darja Kalajdzievska from Winnipeg, Canada, present a paper "Estimation of call prices for some stochastic volatility models". The authors develop a theory to obtain the closed-form expressions of conditional characteristic functions for option pricing for some stochastic volatility models, based on partial differentiation equation. They compare the option prices derived from their method and from the recursive method introduced by some other works. Based on their investigations, the authors conclude that the proposed method significantly reduces the computation time by avoiding the recursive process.

Prof. Rabindra Nath Das from Burdwan University, India, introduces his research "On estimating the optimal process parameters in quality engineering using generalized linear models approach". The most important problem in an industrial process is to predict the operating condition that optimizes a response of interest and simultaneously minimizes the process variability. A modern quality engineering dual response surface (DRS) approach was introduced to achieve this goal. Based on two real examples, this article illustrates how the generalized linear models approach can be used for this aim. Moreover, the paper identifies some drawbacks of the DRS approach which could be misleading in estimation in the optimal level combinations.

Profs. Manana Janiashvili, Nodar Jibladze and Teimuraz Matcharashvili from Tbilisi, Georgia, together with Prof. Alexander Topchishvili, from Marburg, Germany, present a paper "Investigation of dynamical characteristics of blood pressure and heart rate variation in different blood pressure categories". They investigate variation of blood pressure and heart rate characteristics of patients groups from different blood pressure categories, and analyze dynamical features of considered data sets by means of power spectrum regression, detrended fluctuation analysis and multifractal detrended fluctuation analysis. The team compared dynamical characteristics of analyzed data in different blood pressure categories. Based on the analysis results the authors showed that scaling features of considered data sets are different in different blood pressure categories. The researchers found out that by their dynamical features normal and high normal categories are different for all considered physiological characteristics.

Another team of authors leaded by Prof. Vladislav Zhukovskiy, Moscow, and Sergey Sachkov, Orekhovo-Zuevo, Russia, accompanied by Prof. Alexander Topchishvili, presents a paper "Application of probability measures to the existence problem of Berge-Vaisman guaranteed equilibrium". The authors formalize a guaranteed solution notion 
for a non-cooperative game of $n$ persons under uncertainty. They obtain existence conditions for the guaranteed solution in the class of mixed strategies (probability measures) and prove existence of such solution in mixed strategies under standard (for the mathematical game theory) restrictions, such as continuity of payoff functions and compactness of the sets of players' strategies. The researchers use a new method for construction of guaranteed solutions in pure and mixed strategies by reducing construction of a guaranteed solution to construction of a saddle point of a special convolution of the payoff functions.

Profs. Nodar Jibladze and Alexander Topchishvili present the paper "Monte Carlo application and gradient appliance for solving large scale linear programming problems: essence and laboriousness". They propose two iterative algorithms for solving large scale linear programming problems: the first one is based on the gravitation centers method, which in its turn widely uses Monte Carlo statistical test method, and the second one is realized on the basis of the constant step gradient method. Though the developed iterative algorithms are approximate, the authors recommend applying them when solving large scale linear problems, as they are characterized by simplicity of the algorithm and the program, high-speed performance, etc. The authors present some computational results and they assess the both algorithms with respect to a high-speed performance criterion and compare their high-speed performance with a simplex method.

Dr. Jasdev Bhatti together with Prof. Ashok K Chitkara and Dr. Mohit Kumar Kakkar from Punjab, India, present the paper "Stochastic analysis of parallel system with two discrete failures". They analyze two identical parallel units by using discrete distribution and regenerative point technique. They show that system reliability increases with increase in the rate of repair mechanism and decreases with a higher failure rate. It is important for a company following this system to provide better repair facilities to get more profit. The obtained results help to identify the inspection policy for repairs of different kind of failures, and assist to satisfy increasing development of highprecision technologies.

Prof. Galyna Dolenko together with Sviatoslav Lobach from Kyiv, Ukraine, present their paper "System and statistical approach of analysis and forecasting terrorist activity". They consider statistical analysis and prediction of the intervals between terrorist attacks. This study can help to predict next terrorist activities and consider counter-terrorism action plan. Proposed methodology has been illustrated on the examples of terrorist activities in Afghanistan, Algeria, India, Iraq, and the U.S.A on the statistical data for 2000-2010.

The problems discussed in the current issue enrich both statistical and operations research theoretical modeling and their practical applications in a wide variety of problems of modern science, business, and life.

\section{Dr. Stan Lipovetsky \\ MASA Co-Editor-in-Chief Minneapolis, USA}

Dr. Alexander Topchishvili Guest Editor for MASA current special issue Marburg, Germany 